See discussions, stats, and author profiles for this publication at: https://www.researchgate.net/publication/262959474

\title{
Basis properties of a spectral problem with a spectral parameter in the boundary condition (Russian)
}

Article in Doklady Mathematics · October 2006

DOI: 10.1134/S106456240606024X

CITATIONS

20

2 authors:

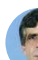

No. Nazim B. Kerimov

Mersin University,Mersin, Turkey

48 PUBLICATIONS 516 CITATIONS

SEE PROFILE

Some of the authors of this publication are also working on these related projects:

Project Sturm-Liouville View project
READS

56

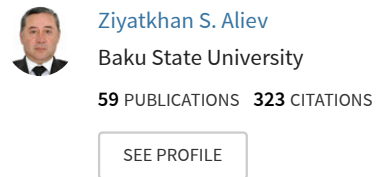




\title{
Some Spectral Properties of a Boundary Value Problem with a Spectral Parameter in the Boundary Condition
}

\author{
N. B. Kerimov ${ }^{a}$ and Z. S. Aliev ${ }^{b}$ \\ Presented by Academician V.A. Il'in June 25, 2006
}

Received May 23, 2006

DOI: $10.1134 /$ S106456240606024X

Consider the eigenvalue problem

$$
\begin{gathered}
\left.\left(p(x) y^{\prime \prime}\right)\right)^{\prime \prime}-\left(q(x) y^{\prime}\right)^{\prime}=\lambda \rho(x) y, \quad 0<x<l, \\
y^{\prime}(0) \cos \alpha-\left(p y^{\prime \prime}\right)(0) \sin \alpha=0, \\
y(0) \cos \beta+T y(0) \sin \beta=0, \\
y^{\prime}(l) \cos \gamma+\left(p y^{\prime \prime}\right)(l) \sin \gamma=0, \\
(a \lambda+b) y(l)-(c \lambda+d) T y(l)=0,
\end{gathered}
$$

where $T y \equiv\left(p y^{\prime \prime}\right)^{\prime}-q y^{\prime} ; \lambda$ is the spectral parameter; the functions $p(x), q(x)$, and $\rho(x)$ are strictly positive and continuous on $[0, l] ; p(x)$ has an absolutely continuous derivative; $q(x)$ is absolutely continuous on $[0, l]$; and $\alpha, \beta, \gamma, a, b, c$, and $d$ are real constants with $0 \leq \alpha, \beta$, $\gamma \leq \frac{\pi}{2}$.

Such problems are encountered in mechanics (see, e.g., [1-6]). If $q(x)$ in Eq. (1) is nonnegative and strictly decreasing on $[0, l]$ and boundary conditions (2) are such that $\alpha=\beta=0, \gamma=\frac{\pi}{2}, a=-1, b=c=0, d=1$, and $l=1$, then boundary value problem (1), (2) describes the transverse vibrations of a vertical (inhomogeneous) rod with a clamped upper end and a load attached to the lower end and with the elastic reactions to bending and tension taken into account [1]. In the case of a homogeneous rod, when $p(x) \equiv \eta_{0}=$ const, $q(x)=k_{0}(1-x)+1$, and $\rho(x) \equiv k_{0}=$ const for $x \in[0,1]$, this problem was described in [2] and was studied in [3], where it was shown that the first eigenvalue is positive and simple. In [1] it was proved that the properties of eigenfrequencies and the corresponding harmonics are quite similar to the spectral properties of a classical string as described by Sturm. If $p(x) \equiv 1$ and $q(x) \equiv 0$ for $x \in[0, l]$ in Eq. (1)

\footnotetext{
${ }^{a}$ Institute of Mathematics and Mechanics, National Academy of Sciences of Azerbaijan, Baku, Azerbaijan

${ }^{b}$ Baku State University, Baku, Azerbaijan
}

and if boundary conditions (2) are such that $\alpha=\beta=0$, $\gamma=\frac{\pi}{2}, a=-M(M=\mathrm{const}>0), b=c=0$, and $d=1$, then the problem corresponds to an inhomogeneous rod with an attached mass $M$. In this case, upper and lower bounds for the first eigenvalue were obtained in [4].

In what follows, it is assumed that

$$
\sigma=b c-a d>0 \text {. }
$$

In this paper, we study the properties of eigenvalues of boundary value problem (1), (2) and the oscillation properties of its eigenfunctions.

\section{SOME AUXILIARY RESULTS}

To study the oscillation properties of eigenfunctions of problem (1), (2), we use the Prüfer-type transformation $[7,8]$

$$
\begin{gathered}
y(x)=r(x) \sin \psi(x) \cos \theta(x), \\
y^{\prime}(x)=r(x) \cos \psi(x) \sin \varphi(x), \\
\left(p y^{\prime \prime}\right)(x)=r(x) \cos \psi(x) \cos \varphi(x), \\
T y(x)=r(x) \sin \psi(x) \sin \theta(x) .
\end{gathered}
$$
form

Equation (1) can be written in the equivalent matrix

$$
U^{\prime}=M U
$$

where

$$
U=\left(\begin{array}{c}
y \\
y^{\prime} \\
p y^{\prime \prime} \\
T y
\end{array}\right), \quad M=\left(\begin{array}{cccc}
0 & 1 & 0 & 0 \\
0 & 0 & \frac{1}{p} & 0 \\
0 & q & 0 & 1 \\
\lambda \rho & 0 & 0 & 0
\end{array}\right) .
$$


Setting $w(x)=\cot \psi(x)$ and applying (4) to (5), we obtain a system of first-order differential equations for $r, w, \theta$, and $\varphi$ :

$$
\begin{gathered}
r^{\prime}=\left[\sin 2 \psi \sin (\theta+\varphi)+\left(q+\frac{1}{p}\right) \cos ^{2} \psi \sin 2 \varphi\right. \\
\left.+\lambda \rho \sin ^{2} \psi \sin 2 \theta\right] \frac{r}{2} \\
w^{\prime}=-w^{2} \cos \theta \sin \varphi+\frac{1}{2}\left(q+\frac{1}{p}\right) w \sin 2 \varphi \\
+\sin \theta \cos \varphi-\frac{\lambda \rho}{2} w \sin 2 \theta \\
\theta^{\prime}=-w \sin \varphi \sin \theta+\lambda \rho \cos ^{2} \theta \\
\varphi^{\prime}=\frac{1}{p} \cos ^{2} \varphi-q \sin ^{2} \varphi-\frac{1}{w} \sin \theta \sin \varphi .
\end{gathered}
$$

The following assertions were stated and proved in [8].

Theorem 1. Let $y(x, \lambda)$ be a nontrivial solution to problem (1), (2a), (2b), (2c) with $\lambda>0$.

Then the Jacobian $J[y]=r^{3} \sin \psi \cos \psi$ of transformation (4) is nonzero for $x \in(0, l)$.

Theorem 2. Let $y(x, \lambda)$ be a nontrivial solution to problem (1), (2a), (2b), (2c) with $\lambda>0$, and let $\theta(x, \lambda)$ and $\varphi(x, \lambda)$ be the corresponding functions in (4).

Then $\theta(0, \lambda)=\beta-\frac{\pi}{2}$ and $\varphi(0, \lambda)=\alpha$, where $\alpha=0$ if $\psi(0, \lambda)=\frac{\pi}{2}$.

Theorem 3. Let $y(x, \lambda)$ be a nontrivial solution to problem (1), (2a), (2b), (2c) with $\lambda>0$, and let $\theta(x, \lambda)$ be the corresponding function in (4).

Then $\theta(l, \lambda)$ is a continuous and strictly increasing function of $\lambda$.

We introduce the boundary condition

$$
y(l) \cos \delta-T y(l) \sin \delta=0, \quad \delta \in\left[0, \frac{\pi}{2}\right] .
$$

Along with problem (1), (2), we consider boundary value problem (1), (2a), (2b), (2c), (2d'). For the latter problem, the oscillation properties of its eigenfunctions corresponding to positive eigenvalues were studied in detail in [8]. In this context, the following two cases were excluded from consideration in [8]: (i) $\alpha=\gamma=0$ and $\beta=\delta=\frac{\pi}{2}$; and (ii) the arbitrary three parameters out of $\alpha, \beta, \gamma$, and $\delta$ are equal to $\frac{\pi}{2}$. Note that, in fact, only the case $\beta=\delta=\frac{\pi}{2}$ is to be excluded.
Theorem 4 [8]. The spectrum of boundary value problem (1), (2a), (2b), (2c), (2d') (except for the case where $\left.\beta=\delta=\frac{\pi}{2}\right)$ consists of an infinite sequence of positive simple eigenvalues $\lambda_{1}^{\delta}<\lambda_{2}^{\delta}<\ldots<\lambda_{n}^{\delta}<\ldots$. Moreover, the eigenfunction $v_{n}^{\delta}(x)$ corresponding to an eigenvalue $\lambda_{n}^{\delta}$ has exactly $n-1$ simple zeros in the interval $(0, l)$, and $T v_{n}^{\delta}(x)$ has exactly $n$ zeros on $[0, l]$.

Remark 1. When $\beta=\delta=\frac{\pi}{2}$, the first eigenvalue of problem (1), (2a), (2b), (2c), (2d') is zero and the corresponding eigenfunction is a constant, and, for $n \geq 2$, Theorem 4 holds true.

\section{BASIC PROPERTIES OF THE SOLUTION TO PROBLEM (1), (2a), (2b), (2c), (2d')}

Lemma 1. For every fixed $\lambda \in \mathbb{C}$, problem (1), (2a), $(2 \mathrm{~b}),(2 \mathrm{c})$ has a unique nontrivial solution $y(x, \lambda)$ up to a constant factor.

Remark 2. Without losing generality, for every fixed $x \in[0, l]$, the function $y(x, \lambda)$ can be assumed to be an entire function of $\lambda$.

Theorem 5. Let $y(x, \lambda)$ be a nontrivial solution to problem (1), (2a), (2b), (2c) and one of the following conditions be satisfied: (i) $\lambda<0$ or (ii) $\lambda=0$ and $\beta \in\left[0, \frac{\pi}{2}\right)$.

Then the Jacobian $J[y]=r^{3} \sin \psi \cos \psi$ of transformation (4) is nonzero for $x \in(0, l)$.

Let $y(x, \lambda)$ be a nontrivial solution to problem (1), (2a), (2b), (2c) and $\lambda \in \mathbb{R} \backslash\{0\}$ or $\lambda=0$ and $\beta \in\left[0, \frac{\pi}{2}\right)$. Assume that $\theta(x, \lambda)$ and $\varphi(x, \lambda)$ are the corresponding functions in (4). Without loss of generality, the initial values of these functions can be specified as follows (see Theorem 2): $\theta(0, \lambda)=\left(\beta-\frac{\pi}{2}\right) \operatorname{sgn} \sin \psi(0, \lambda)$ and $\varphi(0, \lambda)=\alpha$, where $\alpha=0$ if $\psi(0)=\frac{\pi}{2}$.

Obviously, the eigenvalues $\mu_{n}=\lambda_{n}(0)$ and $v_{n}=\lambda_{n}(0)$ $(n \in \mathbb{N})$ of problem (1), (2a), (2b), (2c), (2d') for $\delta=0$ and $\delta=\frac{\pi}{2}$ are the zeros of the entire functions $y(l, \lambda)$ and $T y(l, \lambda)$, respectively. Note that $T y(l, \lambda) / y(l, \lambda)$ is defined for $\lambda \in D \equiv \bigcup_{n=1}^{\infty}\left(\mu_{n-1}, \mu_{n}\right)$, where $\mu_{0}=-\infty$.

DOKLADY MATHEMATICS Vol. 74 No. 32006 
Lemma 2. In each interval $\left(\mu_{n-1}, \mu_{n}\right), n \in \mathbb{N}$, the function $\frac{T y(l, \lambda)}{y(l, \lambda)}$ is continuous and strictly increasing.

Lemma 3. It holds that

$$
\lim _{\lambda \rightarrow-\infty}\left(\frac{T y(l, \lambda)}{y(l, \lambda)}\right)=-\infty
$$

Remark 3. Theorem 4 and Lemmas 2 and 3 imply that, if $\lambda<0$ or $\lambda=0$ and $\beta \in\left[0, \frac{\pi}{2}\right)$, then $\frac{T y(l, \lambda)}{y(l, \lambda)}<$ 0 ; and if $\lambda=0$ and $\beta=\frac{\pi}{2}$, then $T y(l, \lambda)=0$; moreover, $v_{1}<\mu_{1}<v_{2}<\mu_{2}<\ldots$

Denote by $m(\lambda)$ and $s(\lambda)$ the numbers of zeros of $y(x, \lambda)$ and $T y(x, \lambda)$ in the interval $(0, l)$, respectively.

Theorem 6. The following assertions are true:

(a) If $\lambda \in\left(\mu_{n-1}, \mu_{n}\right], n \in \mathbb{N}$, then $m(\lambda)=n-1$.

(b) If $\beta \in[0, \pi / 2)$ and $\lambda \in\left(\mu_{n-1}, \mu_{n}\right], n \in \mathbb{N}$, then $s(\lambda)=n-1$ for $\lambda \in\left(\mu_{n-1}, v_{n}\right]$ and $s(\lambda)=n$ for $\lambda \in\left(v_{n}, \mu_{n}\right]$.

(c) If $\beta=\pi / 2$ and $\lambda \in\left(\mu_{0}, v_{1}\right) \cup\left(v_{1}, \mu_{1}\right]$, then $s(\lambda)=0$, and if $\beta=\pi / 2$ and $\lambda \in\left(\mu_{n-1}, \mu_{n}\right]$ for $n \geq 2$, then $s(\lambda)=$ $n-2$ for $\lambda \in\left(\mu_{n-1}, v_{n}\right]$ and $s(\lambda)=n-1$ for $\lambda \in\left(v_{n}, \mu_{n}\right]$.

The proof of Theorem 6 is based on Theorems 1, 3, and 5 and Lemmas 2 and 3.

\section{MAIN RESULTS}

Lemma 4. The eigenvalues of boundary value problem (1), (2) are real.

Lemma 5. All the eigenvalues of boundary value problem (1), (2) are simple and form at most a countable set without a finite limit point.

When $c \neq 0$, the number $N$ is determined by the inequality $\mu_{N-1}<-\frac{d}{c} \leq \mu_{N}$.

Theorem 7. The eigenvalues of boundary value problem (1), (2) form an infinitely increasing sequence $\lambda_{1}, \lambda_{2}, \ldots, \lambda_{n}, \ldots$ and the corresponding eigenfunctions have the following oscillation properties:

(a) If $c=0$, then the eigenfunction $y_{n}(x)$ corresponding to an eigenvalue $\lambda_{n}$ has exactly $n-1$ simple zeros in the interval $(0, l)$.

(b) If $c \neq 0$, then the eigenfunction $y_{n}(x)$ corresponding to an eigenvalue $\lambda_{n}$ has $n-1$ simple zeros in the interval $(0, l)$ for $n \geq N$ and has exactly $n-2$ simple zeros in this interval for $n>N$.

Proof sketch of Theorem 7. To prove the existence of eigenvalues, it is sufficient to show that the equation

$$
\frac{T y(l, \lambda)}{y(l, \lambda)}=\frac{a \lambda+b}{c \lambda+d}
$$

has a solution.
By Lemma 2, the function $\frac{T y(l, \lambda)}{y(l, \lambda)}$ is continuous and strictly increasing in the interval $\left(\mu_{n-1}, \mu_{n}\right), n \in \mathbb{N}$. Lemma 3 and the equality $y\left(l, \mu_{n}\right)=0, n \in \mathbb{N}$ imply that

$$
\lim _{\lambda \rightarrow \mu_{n-1}+0} \frac{T y(l, \lambda)}{y(l, \lambda)}=-\infty, \quad \lim _{\lambda \rightarrow \mu_{n}-0} \frac{T y(l, \lambda)}{y(l, \lambda)}=+\infty .
$$

For $\frac{(a \lambda+b)}{(c \lambda+d)}$, we have $\left(\frac{a \lambda+b}{c \lambda+d}\right)^{\prime}=-\frac{\sigma}{(c \lambda+d)^{2}}$. Since $\sigma>0$, we conclude that $\frac{(a \lambda+b)}{(c \lambda+d)}$ is strictly decreasing in the interval $(-\infty,+\infty)$ if $c=0$. If $c \neq 0$, the function $\frac{(a \lambda+b)}{(c \lambda+d)}$ is strictly decreasing in each of the intervals $\left(-\infty,-\frac{d}{c}\right)$ and $\left(-\frac{d}{c},+\infty\right) ;$ moreover,

$$
\lim _{\lambda \rightarrow-\frac{d}{c}-0} \frac{a \lambda+b}{c \lambda+d}=-\infty, \quad \lim _{\lambda \rightarrow-\frac{d}{c}+0} \frac{a \lambda+b}{c \lambda+d}=+\infty .
$$

Let $c=0$, or let $c \neq 0$ and $-\frac{d}{c} \notin\left(\mu_{n-1}, \mu_{n}\right]$. The unique solution $\lambda_{n}^{*} \in\left(\mu_{n-1}, \mu_{n}\right)$ to Eq. (6) with $c=0$ or $c \neq 0$ and $n<N$ is the $n$th eigenvalue of boundary value problem (1), (2).

Let $c \neq 0$ and $-\frac{d}{c} \in\left(\mu_{N-1}, \mu_{N}\right)$. In each of the intervals $\left(\mu_{N-1},-\frac{d}{c}\right)$ and $\left(-\frac{d}{c}, \mu_{N}\right)$, Eq. (6) has the unique solution $\lambda_{N}$ and $\lambda_{N+1}$, respectively.

When $c \neq 0$ and $-\frac{d}{c}=\mu_{N}$, we have $\lambda_{N} \in\left(\mu_{N-1}, \mu_{N}\right)$ and $\lambda_{N+1}=\mu_{N}$.

When $c \neq 0$ and $n>N$, the unique solution $\lambda_{n}^{*}$ to Eq. (6) in $\left(\mu_{n-1}, \mu_{n}\right]$ is the $(n+1)$ st eigenvalue of problem (1), (2).

Assertions (a) and (b) in Theorem 7 follow directly from Theorem 6.

Let $\tau_{0}$ be defined as $\tau_{0}=\frac{T y(l, 0)}{y(l, 0)}$.

Theorem 8. Let $\left\{\lambda_{n}\right\}_{n=1}^{\infty}$ be the eigenvalues of boundary value problem (1), (2).

Then $\lambda_{n}>0$ for $n \geq 3$. Moreover, the following assertions hold true.

(a) If $c d>0$ and $\frac{b}{d}<\tau_{0}$, then $\lambda_{1}<\lambda_{2}<0$.

(b) If $c d>0$ and $\frac{b}{d}=\tau_{0}$, then $\lambda_{1}<\lambda_{2}=0$.

(c) If $c d>0$ and $\frac{b}{d}>\tau_{0}$, then $\lambda_{1}<0<\lambda_{2}$. 
(d) If $d=0$, then $\lambda_{1}<0<\lambda_{2}$.

(e) If $c d<0$ and $\frac{b}{d}<\tau_{0}$, then $\lambda_{1}<0<\lambda_{2}$.

(f) If $c d<0$ and $\frac{b}{d}=\tau_{0}$, then $\lambda_{1}=0$.

(g) If $c d<0$ and $\frac{b}{d}>\tau_{0}$, then $\lambda_{1}>0$.

(h) If $c=0$ and $\frac{b}{d}<\tau_{0}$, then $\lambda_{1}<0<\lambda_{2}$.

(i) If $c=0$ and $\frac{b}{d}=\tau_{0}$, then $\lambda_{1}=0$.

(j) If $c=0$ and $\frac{b}{d}>\tau_{0}$, then $\lambda_{1}>0$.

The proof of Theorem 8 is based on Lemmas 2, 3 and Theorem 7.

Remark 4. Note that the number of zeros of $T y_{n}(x)$, $n \in \mathbb{N}$ can be determined in the same manner. For example, let $c d>0$ and $\frac{b}{d}<\tau_{0}$. By Theorem 8 , we have $\lambda_{1}<\lambda_{2}<0$ and $\lambda_{n}>0$ for $n \geq 3$. Theorem 7 implies that $\lambda_{n} \in\left(\mu_{n-2}, v_{n-1}\right)$ for $n \geq 3$. Then, by Theorem 6 , we obtain the following results:

(i) If $\beta \in\left[0, \frac{\pi}{2}\right)$, then $s\left(\lambda_{1}\right)=0$ and $s\left(\lambda_{n}\right)=n-2$.

(ii) If $\beta=\frac{\pi}{2}$, then $s\left(\lambda_{1}\right)=s\left(\lambda_{2}\right)=0$ and $s\left(\lambda_{n}\right)=n-3$ for $n \geq 3$.

Cases (b)-(j) can be considered in the same manner (see Theorem 8).

\section{REFERENCES}

1. S. V. Meleshko and Yu. V. Pokornyi, Differ. Uravn. 23, 1466-1467 (1987).

2. G. H. Handeiman and J. B. Keller, Proceedings of IV US National Congress of Applied Mechanics (New York, 1962), pp. 195-202.

3. H. J. Ahn, Quart. Appl. Math. 39 (1), 109-117 (1981).

4. M. R. Racheva, Dokl. Bolg. Akad. Nauk 54 (11), 23-26 (2001).

5. M. R. Racheva and B. A. Andreev, Comput. Methods Appl. Math. 2 (2), 171-185 (2002).

6. Zh. Amara and A. A. Vladimirov, Izv. Akad. Nauk, Ser. Mat. 68 (4), 3-18 (2004).

7. D. O. Banks and G. J. Kurowski, Trans. Am. Math. Soc. 199, 203-222 (1974).

8. D. O. Banks and G. J. Kurowski, J. Differ. Equations 27 (2), 57-74 (1977). 